\title{
Rapid assessment of infant feeding support to HIV-positive women accessing prevention of mother-to-child transmission services in Kenya, Malawi and Zambia
}

\author{
Mickey Chopra ${ }^{1,2, *}$, Tanya Doherty ${ }^{1,2}$, Saba Mehatru ${ }^{3}$ and Mark Tomlinson ${ }^{1,4}$ \\ ${ }^{1}$ Health Systems Research Unit, Medical Research Council, Francie Van Zyl Drive, Parow, Western Cape 7535, \\ South Africa: ${ }^{2}$ School of Public Health, University of the Western Cape, Cape Town, South Africa: ${ }^{3}$ UNICEF, \\ Eastern and Southern Africa Regional Office, Nutrition Division, Nairobi, Kenya: ${ }^{4}$ Department of Psychology, \\ University of Stellenbosch, Stellenbosch, South Africa
}

Submitted 15 September 2008: Accepted 8 February 2009: First published online 30 April 2009

\begin{abstract}
Objective: The possibility of mother-to-child transmission (MTCT) of HIV through breast-feeding has focused attention on how best to support optimal feeding practices especially in low-resource and high-HIV settings, which characterizes most of sub-Saharan Africa. To identify strategic opportunities to minimize late postnatal HIV transmission, we undertook a review of selected country experiences on HIV and infant feeding, with the aims of documenting progress over the last few years and determining the main challenges and constraints.

Design: Field teams conducted national-level interviews with key informants and visited a total of thirty-six facilities in twenty-one sites across the three countries eighteen facilities in Malawi, eleven in Kenya and seven in Zambia. During these visits interviews were undertaken with key informants such as the district and facility management teams, programme coordinators and health workers.

Setting: A rapid assessment of HIV and infant feeding counselling in Kenya, Malawi and Zambia, undertaken from February to May 2007.

Results: Infant feeding counselling has, until now, been given low priority within programmes aimed at prevention of MTCT (PMTCT) of HIV. This is manifest in the lack of resources - human, financial and time - for infant feeding counselling, leading to widespread misunderstanding of the HIV transmission risks from breastfeeding. It has also resulted in lack of space and time for proper counselling, poor support and supervision, and very weak monitoring and evaluation of infant feeding. Finally, there are very few examples of linkages with community-based infant feeding interventions. However, all three countries have started to revise their feeding policies and strategies and there are signs of increased resources.

Conclusions: In order to sustain this momentum it will be necessary to continue the advocacy with the HIV community and stress the importance of child survival - not just minimization of HIV transmission - and hence the need for integrating MTCT prevention.
\end{abstract}

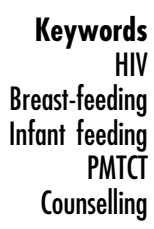

The transmission of HIV through breast milk has provided significant scientific and programmatic challenges in minimizing mother-to-child transmission (MTCT) through prevention of MTCT (PMTCT) programmes. The rate of transmission of HIV infection in breast-fed infants is cumulative and increases with duration of breast-feeding; the estimated cumulative probability of transmission (with antiretroviral prophylaxis at birth) between 4 weeks and 18 months of age is $9 \cdot 3 \%{ }^{(1)}$. Furthermore, approximately $42 \%$ of all HIV infections in infants are attributable to breastfeeding in settings where prolonged breast-feeding is common $^{(1)}$. There is now substantial evidence that investment in infant feeding support can substantially reduce late postnatal transmission even in very resource-poor settings ${ }^{(2-4)}$.

Recent evaluations from routine PMTCT programmes suggest that early MTCT can be significantly reduced through effective peripartum prophylaxis ${ }^{(5,6)}$; however, late postnatal transmission remains disturbingly $\operatorname{high}^{(7,8)}$. A recent $\mathrm{WHO} / \mathrm{UNICEF}$ review of programmatic experience found a number of shortcomings in the coverage and quality of infant feeding counselling and support across most PMTCT programmes ${ }^{(9)}$. A South African study found that poor-quality infant feeding counselling resulted in inappropriate infant feeding choices being made by the mother, 
leading to a threefold increase in risk of infant HIV transmission or death ${ }^{(10)}$. Some commentators have suggested that the HIV epidemic has seriously undermined breastfeeding and child nutrition activities especially in Africa ${ }^{(11)}$. The need to address the reversal of early gains in HIV-free survival due to poor infant feeding practices and to strengthen infant nutrition programmes are now widely recognized as priorities.

In order to identify strategic opportunities to minimize late postnatal HIV transmission, we undertook a review of selected country experiences on HIV and infant feeding. The main objectives of the review were to document the progress of the last few years and determine the main challenges and constraints.

\section{Methods}

Three sub-Saharan African countries highly affected by HIV (Kenya, Malawi and Zambia) were purposively selected for the field work; selection was on the basis that the three countries are approaching national coverage with their PMTCT programmes and had recognized the need to review infant feeding components of the programme. The field work was carried out between February and May, 2007. We reviewed recent relevant policy documents, training materials, reports and minutes of interagency coordination committee meetings. Field teams conducted national-level interviews with key informants and visited a total of thirty-six facilities in twenty-one sites across the three countries - eighteen facilities in Malawi, eleven in Kenya and seven in Zambia. Sites were purposively selected to reflect the different settings (urban and rural) and programme implementers (government $v$. non-governmental organizations (NGO) $v$. UN) in each country. During these visits interviews were undertaken with key informants such as the district and facility management teams, programme coordinators and health workers. The visits aimed to determine where HIV and infant feeding counselling and support had been implemented; review reporting and monitoring mechanisms in facilities to determine coverage of HIV and infant feeding counselling support; and explore health workers' perceptions of the use of primary healthcare infrastructure to support HIV-positive mothers with counselling on HIV and infant feeding.

Two semi-structured data collection tools were developed, one for interviews with national-level stakeholders and one for field-site interviews, and an observation tool was developed for observations of facilities and outreach posts. Sites for field visits were purposively selected to represent a range of areas that were performing well and performing poorly and included health facilities, clinics, outreach posts, schools and mobile units. Data analysis involved reviews of national policy documents and reports, and content analysis of interview transcripts to identify commonly occurring themes.

\section{Results}

According to the responses from interviews with national and site managers, the Ministry of Health and PMTCT implementation partners in all three countries welcomed a focus on the infant feeding component of PMTCT programmes, but their responses along with the facility assessments raised some critical issues.

\section{Policy making}

Infant feeding was low on the agenda when PMTCT programmes were initiated. One respondent stated:

Infant feeding is so invisible because when it comes to health prioritization it depends to a great degree on who is pushing what agenda. What becomes prioritized depends to a great extent on whether it is being pushed by (for example) an obstetrician, gynaecologist or nutritionist. It is about who is pushing harder and shouting the loudest.

Unfortunately, nutrition did not have enough champions in these forums until recently. However, signs are emerging that leadership is developing and key staff are responding positively to the challenges. For example, the Kenyan National AIDS and STI Control Programme (NASCOP) used to have just one nutritionist but now employs fifty nutritionists specifically to be involved in HIV service delivery points. In Zambia, the national PMTCT technical working group, which operates under the auspices of the National AIDS Council, is sympathetic to infant feeding issues but it is only an advisory group. HIV and Infant Feeding falls under the 'feeding in difficult circumstances' component of the Infant and Young Child Feeding programme of the Nutrition Unit of the Ministry of Health. In Malawi, the National PMTCT Task Force is the coordinating body for MTCT prevention, with representatives from all agencies that implement services. In addition, infant feeding is handled by the nutrition unit, which has an infant feeding coordinator. This person works closely with the PMTCT coordinator to support HIV and Infant Feeding activities, and is a member of the national PMTCT working group.

Yet, there still remains a huge gap in the level of political support that nutrition or infant feeding is able to attract compared with the other components of the PMTCT programme. Respondents across Kenya and Zambia agreed that nutrition is the 'poor cousin' in the Ministry of Health and that its position has become weaker in the last decade. For example, in Zambia the National Food and Nutrition Council was a strong and independent group that advised the government on all nutrition issues. However, it has recently suffered significant financial cutbacks and is not able to perform its role to the same extent. The Ministry of Health has only two nutritionists at the central level and no nutritionist at the provincial level. These power imbalances mean that 


\section{Box 1}

\section{HIV and breast-feeding decline}

Nutritionists across all three countries stated that the HIV epidemic has significantly reduced investments in nutrition in general and breast-feeding in particular. This is most directly felt in one important breast-feeding initiative: the Baby-Friendly Hospital Initiative (BFHI).

- Kenya. The number of baby-friendly hospitals fell from over 600 in 1996 to fewer than six in 2003. No formal assessments have taken place for at least the last three years. However, signs of change are emerging, with a number of institutions undergoing self-assessments before being reassessed externally.

- Malawi. The mid- to late-1990s saw an explosion in BFHI and breast-feeding promotion activities, with a corresponding increase in exclusive breast-feeding rates. However, respondents reported that confusion over HIV and breast-feeding had significantly diminished BFHI activities. They are now being prioritized again.

- Zambia. The BFHI began in 1997 with accreditation of forty-six institutions. According to the National Food and Nutrition Commission, it was working well, but the advent of HIV and confusion over infant feeding methods led to significant uncertainty, and the initiative faltered. No BFHI monitoring has taken place in many years. But UNICEF has trained fifteen people on the revised 2006 materials and tools, which incorporate HIV issues, and plans call for extending the initiative into two provinces in 2007. The Commission believes that a baby-friendly hospital is more likely to support HIV-positive women.

the HIV/AIDS division has largely driven PMTCT policies and protocols, and hence training curricula.

Senior officials in the HIV and PMTCT programmes cited the support of UN agencies for formula feeding at selected pilot sites during the 1990s and the subsequent change in policy as an important reason for their confusion around optimal infant feeding policy.* According to one senior respondent:

There was then a significant amount of confusion over exclusive breast-feeding, especially as we were also trying to scale up the Baby-Friendly Hospital Initiative... I would say there was open warfare amongst recipients regarding who was receiving formula and who was not.

This does seem to also have translated into a sharp slowdown in important breast-feeding interventions in the countries (Box 1).

This also meant that nutrition policies said very little about HIV and infant feeding during that time. For example, the Zambian National Food and Nutrition Policy of 2003 mentions it only once, although Zambia later developed the Infant and Young Child Feeding Operational Strategy which elaborated the HIV and Infant Feeding strategies.

\section{Resource mobilization}

The divisions of nutrition in the ministries of health were consistently weak, particularly in financial resources. For

* It should be noted that at the time of the implementation of the pilot PMTCT interventions by UNICEF the evidence from drug trials had been based upon women who did not breast-feed their infants. Based on the experience gained from the PMTCT pilot project, UNICEF developed its policy in 2002 to end the procurement and distribution of free formula as part of its support to PMTCT. The arguments against formula provision are detailed in the UNICEF operational guidance note on infant feeding and MTCT of HIV. example, in one country, donor funding constitutes about $20 \%$ of total ministry funding. Yet the division of nutrition was dependent on donor funding for 80 to $90 \%$ of its budget - a clear sign that it was not being prioritized by the ministry. In another country, the total monthly budget for the whole nutrition department was one-third of the cost of training thirty people for five days. The nutrition department was weak in negotiating skills, making it hard to obtain funds from PMTCT units to support infant feeding activities.

More encouragingly, a number of important donors stated they were beginning to focus more on the challenges of infant feeding and were setting aside substantially increased funds for it in the coming financial year. Kenya, for example, has committed resources from the Global Fund to employ fifty nutritionists, who are now stationed across the country. Malawi has also raised awareness of the importance of nutrition. A key respondent said that the budget for nutrition had doubled in the last few years to about $1 \%$ of the Ministry of Health's budget.

\section{Guidelines and protocols}

At the regional and global levels, there is increasingly strong advocacy in support of strengthening the HIV and Infant Feeding components of the PMTCT programmes. To this end, the present UN recommendation that HIVpositive pregnant women should receive individual counselling on various feeding options, including the health benefits and risks of breast-feeding and alternative feeding, forms the basis of the infant feeding sections of PMTCT guidelines and protocols in all three countries. However, more work needs to be done. The criteria of affordability, feasibility, acceptability, safety and sustainability are outlined but local adaptation and examples of what is meant by these terms were not found. The documents include little or no mention of issues which 


\section{Box 2}

\section{Confusing messages on HIV and breast-feeding}

Interviews with medical officers and senior nurses particularly in two out of the three countries revealed widespread unease and uncertainty around the risks of breast-feeding, rapid weaning and the interpretation of AFASS criteria (to determine whether breast-feeding is acceptable, feasible, affordable, sustainable and safe). Most concerning was the resulting discordance in advice given to HIV-positive mothers:

We offer PCR infant testing at six weeks and then tell mothers of HIV-negative infants to stop breast-feeding. (Kenyan medical officer, Nairobi)

We only offer PCR testing two weeks after breast-feeding has stopped. (Kenyan medical officer, Eldoret)

We tell all mothers to stop breast-feeding at six months. (Zambian medical officer, Ndola)

We only stop mothers from breast-feeding when it is safe to do so. (Zambian medical officer, Lusaka)

still remain critical, such as: cessation of breast-feeding and what should be advised for the non-breast-fed child; the type of infant feeding counselling that should be offered now that many infants are receiving early HIV testing by PCR at six weeks; and advice on maternal nutrition, especially for HIV-positive lactating mothers.

Senior officials expressed great concern about these issues and requested urgent assistance in resolving them. This uncertainty and confusion manifests itself at the facility level through a great deal of variability in the interpretation of guidelines (Box 2). Some donors and NGO have started to implement algorithms and job-aids to assist in counselling sessions. But the team did not find consistent use of such aids, nor was there any evidence of their effectiveness (though the WHO is currently sponsoring an evaluation in Zambia). The team consistently found that nearly all health workers, including doctors and nurses, massively overestimated the risk of HIV transmission through breast-feeding or did not know the risk. Most health workers providing infant feeding counselling as part of the PMTCT programme report a need for more training on infant feeding (described as 'breast-feeding course'). Most said they do not receive supervision, but feel supported by colleagues. The workload of many is high - up to twenty clients counselled on a daily basis - and many described their working environment as stressful. The lack of capacity and supervision leading to poor quality of infant feeding counselling was especially worrying in the sites that offered free formula as part of the package.

We did not find any examples of detailed management guidelines concerning the roles and responsibilities or priority setting that would assist district and facility managers in planning for integration of infant feeding counselling into routine services. The consequences of this absence were manifested in various ways, including lack of HIV and infant feeding supervisory tools, haphazard human resource planning in terms of who was sent for training, complex monitoring and evaluation systems, and duplication of training.
Despite these challenges, there were encouraging signs that infant and young child feeding leaders are beginning to reassert the role of optimal infant feeding for reducing MTCT and improving child survival. In particular, the three countries have adopted and adapted the Global Strategy for Infant and Young Child Feeding ${ }^{(12)}$ into policies and guidelines that explicitly address HIV and infant feeding issues within a broader nutrition framework.

\section{Capacity building}

In all three countries comprehensive PMTCT courses had been developed based on the Generic Training Package of WHO and the Centers for Disease Control and Prevention.

Zambia offers a twelve-day course, in Kenya it has been cut down to six days but in both countries only four hours are spent on infant feeding counselling, with no time for practical simulation. The content covered in this limited time is very broad. Malawi has a ten-day course, of which about three days are given to infant feeding counselling, including some time for practical simulations.

Analysis of the PMTCT curricula in all three countries also revealed a strong bias against breast-feeding. The curricula clearly outline and quantify the risk of MTCT from breast-feeding - in the case of Kenya, overestimating this risk by a factor of three. However, the risk of excess morbidity or mortality from replacement feeding is not at all quantified. Instead it is mentioned as a potential disadvantage, next to the possible advantages of replacement feeding. Exclusive breast-feeding is given the same amount of time and emphasis as other modes of feeding such as modification of cow's milk, heat treatment and replacement feeding. The curricula do not cover common challenges in supporting exclusive breastfeeding, such as responding to the mother's perception that her breast milk is not sufficient.

Senior nutritionists reported that they had attempted to participate in developing the PMTCT curriculum, but clinicians had dominated the process and nutritionists had been marginalized. In one country extensive lobbying 
had at least succeeded in adding guidelines (as appendices) to the training and participant manuals about how to modify cow's milk, heat breast milk and a number of other components. Unfortunately when the manuals were printed these appendices were left out, awaiting revisions of the materials based on updated national infant and young child feeding guidelines. Kenya is now revising its PMTCT curriculum, and senior managers have promised to involve the nutritionist more and to give more time to infant feeding.

A previous UNICEF outcome assessment of Kenya's course found no difference in health workers' knowledge of the risks of MTCT between those who had been trained and those who had not - knowledge was uniformly poor in both groups ${ }^{(13)}$. The team could find no systematic evaluation of the courses in Malawi or Zambia.

Nutritionists in all three countries are attempting to address the gap in counselling capacity by implementing additional HIV and infant feeding courses. Kenya is establishing regional lactation management centres in provincial hospitals and training nurses in the 40-hour lactation course. But there is little funding from the Ministry of Health, and donor funding still remains limited. Zambia was hoping to finalize a curriculum for a five-day integrated course on Infant and Young Child Feeding by the end of April 2007. No resources for conducting this training had yet been secured. Malawi is also planning a more intensive five-day HIV and Infant Feeding course. Malawi, on the other hand, has made a concerted, successful effort to incorporate HIV and infant feeding issues into existing child health and nutrition courses such as Integrated Management of Childhood Illness and BabyFriendly Hospital Initiative training and has included them as part of the Essential Nutrition Actions framework.

In a majority of the site visits, it was the medical officer, either at facility or district level, who seemed to have the greatest influence on the tone and focus of infant feeding counselling. Therefore, the ongoing integrated course on Infant and Young Child Feeding, including HIV and Infant Feeding counselling, plus periodic updates on HIV and Infant Feeding, need to especially target medical practitioners in all three countries.

\section{Monitoring and evaluation}

A focus on outputs, such as numbers of clients tested and numbers on treatment, has been an important feature of the successful scaling up of PMTCT across the region. This approach is now being used to scale up the number of infants on treatment. But none of the three countries has clear output or outcome indicators related to infant feeding counselling, which is a reflection of the global reporting format for the PMTCT report card. To this end, the Zambian National PMTCT and Pediatric Report Card (January-December 2005) has no mention of infant feeding. However, Kenya has taken the initiative to strengthen key indicators on infant feeding in national monitoring tools for HIV service provision at PMTCT facilities.

The number of women receiving infant feeding counselling and the proportion of women selecting breastfeeding were two common indicators collected. However, senior managers and donors do not find these indicators very informative. First, they are poorly collected, and second, they give no sense of the quality and hence effectiveness of this component. Important outcome indicators such as proportion of mothers exclusively breast-feeding were not being routinely collected because they are very difficult to perform and likely to give poorquality data. Therefore, efforts are needed to collect such information through periodic surveys.

In all three countries, infant health cards are being revised to explicitly allow identification of maternal HIV status and to record infant feeding practices. Sharing of best practices in this regard would be beneficial. Finally, the team consistently found that performance assessment tools at the district and primary care level made minimal reference to infant feeding, thus requiring further attention.

\section{Discussion}

We found a number of shortcomings that explain the present weakness in infant feeding counselling. However, signs are appearing that countries are developing and responding positively to the challenges. For example, all three countries have started to revise their infant and young child feeding policies following the lead of and with support from UN agencies. This has led to the establishment of strong working groups that have incorporated HIV and infant feeding into the broader infant feeding strategy.

There are some limitations to this study. The purposive sampling of countries that recognized the need for such a review limits the generalizability of these findings. Limited time and resources did not allow for systematic random sampling of sites across the whole country and this may have introduced a selection bias. However, there was consistency across national sites in the findings reported here.

In order to sustain this momentum it will be necessary to continue the advocacy with the HIV community of the importance in investing in infant feeding within the context of HIV programmes. Another key message needs to be the wider recognition and prioritization of child survival - not just minimization of HIV transmission - and hence the need for integrating MTCT prevention. This presents opportunities to reconsider the roles, responsibilities and capacities of health workers and counsellors, especially with respect to strengthening infant feeding counselling and redesigning maternal and child health cards. Finally, improving linkages with and strengthening community-based support systems for child health will benefit not just HIV-exposed infants but also the greater majority of non-exposed children. 


\section{Acknowledgements}

This study was funded by UNICEF, Eastern and Southern Africa Regional Office. We have no conflicts of interest to report. M.C., T.D. and M.T. designed the study and developed the data collection tools. T.D. and M.T. trained and supervised the data collection. All authors contributed to the data analysis and writing of the manuscript.

\section{References}

1. Coutsoudis A, Dabis F, Fawzi W et al. (2004) Late postnatal transmission of HIV-1 in breast-fed children: an individual patient data meta-analysis. J Infect Dis 189, 2154-2166.

2. Coovadia HM, Rollins NC, Bland RM, Little K, Coutsoudis A, Bennish ML \& Newell ML (2007) Mother-to-child transmission of HIV-1 infection during exclusive breastfeeding in the first 6 months of life: an intervention cohort study. Lancet 369, 1107-1116.

3. Iliff PJ, Piwoz EG, Tavengwa NV, Zunguza CD, Marinda ET, Nathoo KJ, Moulton LH, Ward BJ \& Humphrey JH; ZVITAMBO study group (2005) Early exclusive breastfeeding reduces the risk of postnatal HIV-1 transmission and increases HIV-free survival. AIDS 19, 699-708.

4. Kuhn L, Sinkala M, Kankasa C et al. (2007) High uptake of exclusive breastfeeding and reduced early post-natal HIV transmission. PLOS ONE 2, e1363.

5. Coetzee D, Hilderbrand K, Boulle A, Draper B, Abdullah F \& Goemaere E (2005) Effectiveness of the first districtwide programme for the prevention of mother-to-child transmission of HIV in South Africa. Bull World Health Organ 83, 489-494.
6. Colvin M, Chopra M, Doherty T, Jackson D, Levin J, Willumsen J, Goga A \& Moodley P; Good Start Study Group (2007) Operational effectiveness of single-dose nevirapine in preventing mother-to-child transmission of HIV. Bull World Health Organ 85, 466-473.

7. Jackson DJ, Chopra M, Doherty TM, Colvin MS, Levin JB, Willumsen JF, Goga AE \& Moodley P; for the Good Start Study Group (2007) Operational effectiveness and 36 week HIV-free survival in the South African programme to prevent mother-to-child transmission of HIV-1. AIDS 21, 509-516.

8. Quaghebeur A, Mutunga L, Mwanyumba F, Mandaliya K, Verhofstede C \& Temmerman M (2004) Low efficacy of nevirapine (HIVNET) in preventing perinatal HIV-1 transmission in a real-life situation. AIDS 18, 1854-1856.

9. World Health Organization/UNICEF (2007) HIV and Infant Feeding: New Evidence and Programmatic Experience. Report of a Technical Consultation held on behalf of the Inter-agency Task Team (IATT) on Prevention of HIV Infections in Pregnant Women, Mothers and their Infants, Geneva, Switzerland, 25-27 October 2006. Geneva: WHO.

10. Doherty T, Chopra M, Jackson D, Goga A, Colvin M \& Persson LA (2007) Effectiveness of the WHO/UNICEF guidelines on infant feeding for HIV-positive women: results from a prospective cohort study in South Africa. AIDS 21, 1791-1797.

11. Kisanga P \& Latham M (2001) Impact of Policy on Breastfeeding and MTCT in Four Countries. Consultant Report for UNICEF. Nairobi: UNICEF/ESARO.

12. World Health Organization/UNICEF (2003) Global Strategy for Infant and Young Child Feeding. Geneva: WHO.

13. Chopra M \& Rollins N (2008) Infant feeding in the time of HIV: assessment of infant feeding policy and programmes in four African countries scaling up prevention of mother to child transmission programmes. Arch Dis Child $\mathbf{9 3}$, 288-291. 